\title{
The Future is Non-cemented Total Knee Arthroplasty: Volume Trends at the Hospital for Special Surgery
}

\author{
Samuel Rodriguez ${ }^{1}\left[\right.$ ( ) Amar S. Ranawat ${ }^{1,2,3}$
}

Received: 21 June 2021 / Accepted: 2 September 2021 / Published online: 2 October 2021

(c) Indian Orthopaedics Association 2021

\begin{abstract}
Introduction The optimal fixation method for total knee arthroplasty (TKA) is still a debate. Cemented fixation has excellent long-term results and is the gold standard. However, longevity in the younger, heavier, and more active population is suboptimal. Cementless TKA offers the opportunity to gain biological fixation and overcome these shortcomings.

Methods This is a retrospective review of all consecutive cementless TKA procedures performed at a single academic institution from 2016 until 2020. Demographics, aseptic revisions, and septic revisions were pulled from the electronic medical record. The number of yearly implanted cementless TKA prosthesis was determined to analyze utilization trends.

Results Eight-hundred and two patients were identified with a mean age of $61.57 \pm 7.78$ years, and a mean of BMI $32.12 \pm 5.98 \mathrm{~kg} / \mathrm{m}^{2}$. The mean time to revision was $12.31 \pm 13.91$ months. There were four septic failures and nine aseptic failures during the study period. Of these nine aseptic failures only five were due to mechanical loosening. There was a yearly linear increase in the use of cementless fixation.

Conclusion Cementless fixation is here to stay, and its use will continue to increase. Early and mid-term outcomes have been excellent thus far. Changing clinical practice takes time but we have already seen this transition take place in total hip arthroplasty. As technology and design continue to evolve, we believe it is a possibility.
\end{abstract}

Keywords Total knee arthroplasty $\cdot$ Cementless $\cdot$ Noncemented $\cdot$ TKA

\section{Introduction}

Total knee arthroplasty (TKA) is one of the most commonly performed elective surgical procedures and its incidence continues to increase [1]. Cemented fixation is the gold standard and has excellent long-term results [2-4]. Furthermore, registry-level data reiterates the market share control and outstanding outcomes achieved with cement fixation, however, despite these outstanding results aseptic loosening remains the most common indication for aseptic revision TKA in the United States [3, 5-8]. Cemented fixation

Samuel Rodriguez

rodriguezsam@hss.edu

Amar S. Ranawat

ranawatamar@hss.edu

1 Hospital for Special Surgery, Adult Reconstruction and Joint Replacement, 535 E70th Street, New York, NY 10021, USA

2 Weill Cornell Medical College, New York, USA

3 NewYork-Presbyterian Hospital, New York, USA longevity is multifactorial, influenced both by surgical technique and patient related factors [9]. The bone-cement interface is vulnerable to failure with cyclical loading and lacks the ability to remodel, these factors contribute to aseptic loosening of cemented components. Nowadays younger, heavier and more active patients are undergoing TKA procedures [10]. There is a higher rate of aseptic loosening in this younger patient cohort, especially for patients under 55 years of age [11]. Therefore, improving implant fixation and longevity remains a priority.

The clinical success of cementless total hip arthroplasty (THA) fixation may have reignited the interest in cementless TKA. Modern cementless TKA implants have improved dramatically due to innovations in technology and design. The use of porous titanium and porous tantalum metal surfaces has led to greater coefficient of friction and a reduction in Young's modulus mismatch between the implant and host bone [12]. Also, modern implants use of biologically active surface coatings such as periapatite and hydroxyapatite. In combination these factors increase the potential for ingrowth by reducing micromotion and increasing 
osteoconductive properties $[13,14]$. Thus, we sought to evaluate (1) the utilization trends at our institution and (2) short-term survivorship.

\section{Materials and Methods}

This is an institutional review board approved retrospective review of a prospectively collected database of all consecutive TKA's performed at a single academic institution from 2016 until 2020. Inclusion criteria were patients age $\geq 18$ years undergoing primary TKA and receiving a cementless knee implant. The number of yearly implanted cementless TKA prosthesis was determined to analyze utilization trends.

A midline skin incision was used on all procedures using a medial parapatellar approach. All patients received the same preoperative antibiotic and postoperative anticoagulation protocols. The same physical therapy protocol was also used consisting of immediate weight bearing with active and passive exercises. Different cementless TKA prosthesis were used during the study period. Patient demographics including age, sex, race, ethnicity, American Society of Anesthesiology (ASA) score, and body mass index (BMI) were collected. Patient surgical variables including primary laterality, year of surgery, revision or not, time to first revision, and septic revision were also collected. The cohort was analyzed for yearly utilization count as the primary outcome.

Continuous variables were presented as mean and standard deviation [SD]. Categorical variables were presented as frequencies and percentages (Table 1). Linear regression was conducted for the yearly utilization of cementless TKA Implants (Fig. 1). Statistical analyses were performed using SAS 9.4 (SAS Institute Inc., Cary, NC) and Microsoft Excel, version 16.48 (Microsoft Corporation, Redmond WA).

\section{Results}

Eight hundred fourteen patients received cementless TKA implants with a mean ( \pm standard deviation [SD]) time to revision of $12.3 \pm 13.9$ months, the mean $( \pm$ SD) age was $61.6 \pm 7.8$ years, and the mean $( \pm \mathrm{SD}) \mathrm{BMI}$ was $32.1 \pm 6.0 \mathrm{~kg} / \mathrm{m}^{2}$. During the study period there were 4 septic revisions ( $0.49 \%$ septic failure rate), leaving 810 patients for potential aseptic failure. There were nine aseptic failures ( $1.11 \%$ aseptic failure rate) with different mechanisms of failure. Of these nine aseptic failures five were due to loosening, only one patient had $\mathrm{BMI}<30$. There was a linear yearly upward trend of cementless TKA prosthesis implanted within the last 5 years at our institution $\left(R^{2}=0.81\right)$, with 2020 being the exception (Fig. 1).

\section{Discussion}

TKA is the treatment of choice when conservative management fails for arthritic knees and cemented fixation is the gold standard, however, there is potential to improve these excellent results. In the United States aseptic loosening remains the most common cause for revision TKA. Moreover, the patient population undergoing TKA is becoming younger, more active, heavier, and living longer. Surgeons are facing the challenge to provide long-lasting constructs to this evolving population. Cementless fixation has the potential to tackle some of these challenges.

Cementless fixation offers the possibility to gain biologic fixation, preserve bone stock, and potentially improve survivorship [15]. Other potential advantages of cementless fixation include shorter surgical time, and less third body debris from loose cement particles [16]. Disadvantages include lack of local antibiotic delivery, the need of good bone quality, and higher implant cost. However, Barrack et al. looked into the total costs of implanting cemented vs cementless prostheses taking into consideration surgical time, implant, cement and associated supplies. In their practice cementless TKA resulted in lower total costs compared to cemented TKA resulting from shorter surgical times and lower cementation supply costs, therefore, implant cost alone should not guide this decision [17].

Cementless TKA use has been increasing in recent years, we found there was a consistent upward trend until 2020. Due to the Covid-19 pandemic elective surgeries were canceled for 4 months at our institution. In addition, after elective surgeries restarted the volume of procedures being performed was still significantly lower than previous years [18]. This explains the interruption in the upward trend of cementless TKA utilization.

Early cementless TKA implants had design flaws leading to early failure. When assessing individual components, the femoral component achieved better results than tibial and patellar components. Femoral failures were related to fatigue fractures particularly in thin areas [16]. Other design flaws include the use of sintered beads (on both surfaces), short pegs, partial porous coatings, poor polyethylene locking mechanisms, and the use of metal backed patellae [19-21]. Lessons were learned, and modern iterations have excellent short and mid-term survivorship rates greater than $90 \%$. Also, excellent clinical and patient-reported outcomes have been reported $[8$, 13, 22-25]. Nam et al. performed a randomized clinical trial using the same implant design, randomizing patient either to cemented or cementless fixation. In this study there were no revisions for aseptic loosening two years post operatively. Also, there was no difference of patient 
Table 1 Demographics and surgical variables

\begin{tabular}{|c|c|c|c|}
\hline \multirow[t]{2}{*}{ Variable } & \multicolumn{3}{|c|}{ Cementless } \\
\hline & Total $N$ & Mean/median & $\mathrm{SD} / \mathrm{IQR}$ \\
\hline Patient age at surgery & & 61.55 & 7.75 \\
\hline Body Mass Index (most recent prior) & & 32.04 & 5.95 \\
\hline Months to first revision & & 12.31 & 13.91 \\
\hline \multirow[t]{2}{*}{ Variable } & \multicolumn{3}{|c|}{ Cementless } \\
\hline & Total $N$ & $N$ & $\mathrm{Col} \%$ \\
\hline Sex & 814 & & \\
\hline Female & & 286 & 35.1 \\
\hline Male & & 528 & 64.9 \\
\hline Race & 814 & & \\
\hline White or Caucasian & & 682 & 83.8 \\
\hline Asian & & 18 & 2.2 \\
\hline Black or African-American & & 57 & 7 \\
\hline Others & & 44 & 5.4 \\
\hline NA & & 13 & 1.6 \\
\hline Hispanic_ethnicity & 814 & & \\
\hline Hispanic & & 48 & 5.9 \\
\hline Not hispanic & & 757 & 93 \\
\hline NA & & 9 & 1.1 \\
\hline Year of DOS & 814 & & \\
\hline 2016 & & 32 & 3.9 \\
\hline 2017 & & 100 & 12.3 \\
\hline 2018 & & 167 & 20.5 \\
\hline 2019 & & 288 & 35.4 \\
\hline 2020 & & 227 & 27.9 \\
\hline ASA score & 814 & & \\
\hline 1 & & 29 & 3.6 \\
\hline 2 & & 669 & 82.2 \\
\hline 3 & & 116 & 14.3 \\
\hline 4 & & & 0 \\
\hline Side (primary surgery) & 814 & & \\
\hline NA & & & 0 \\
\hline Bilateral & & 34 & 4.2 \\
\hline Left & & 359 & 44.1 \\
\hline Right & & 421 & 51.7 \\
\hline Has revision & 814 & & \\
\hline No & & 801 & 98.4 \\
\hline Yes & & 13 & 1.6 \\
\hline Cementless_revision_septic & 814 & & \\
\hline No & & 809 & 99.4 \\
\hline Yes & & 4 & 0.6 \\
\hline Model & 814 & & \\
\hline ATTUNE & & 18 & 2.2 \\
\hline EMPOWR & & 2 & 0.2 \\
\hline PERSONA & & 5 & 0.6 \\
\hline SIGMA & & 135 & 16.6 \\
\hline TRIATHLON & & 654 & 80.3 \\
\hline
\end{tabular}




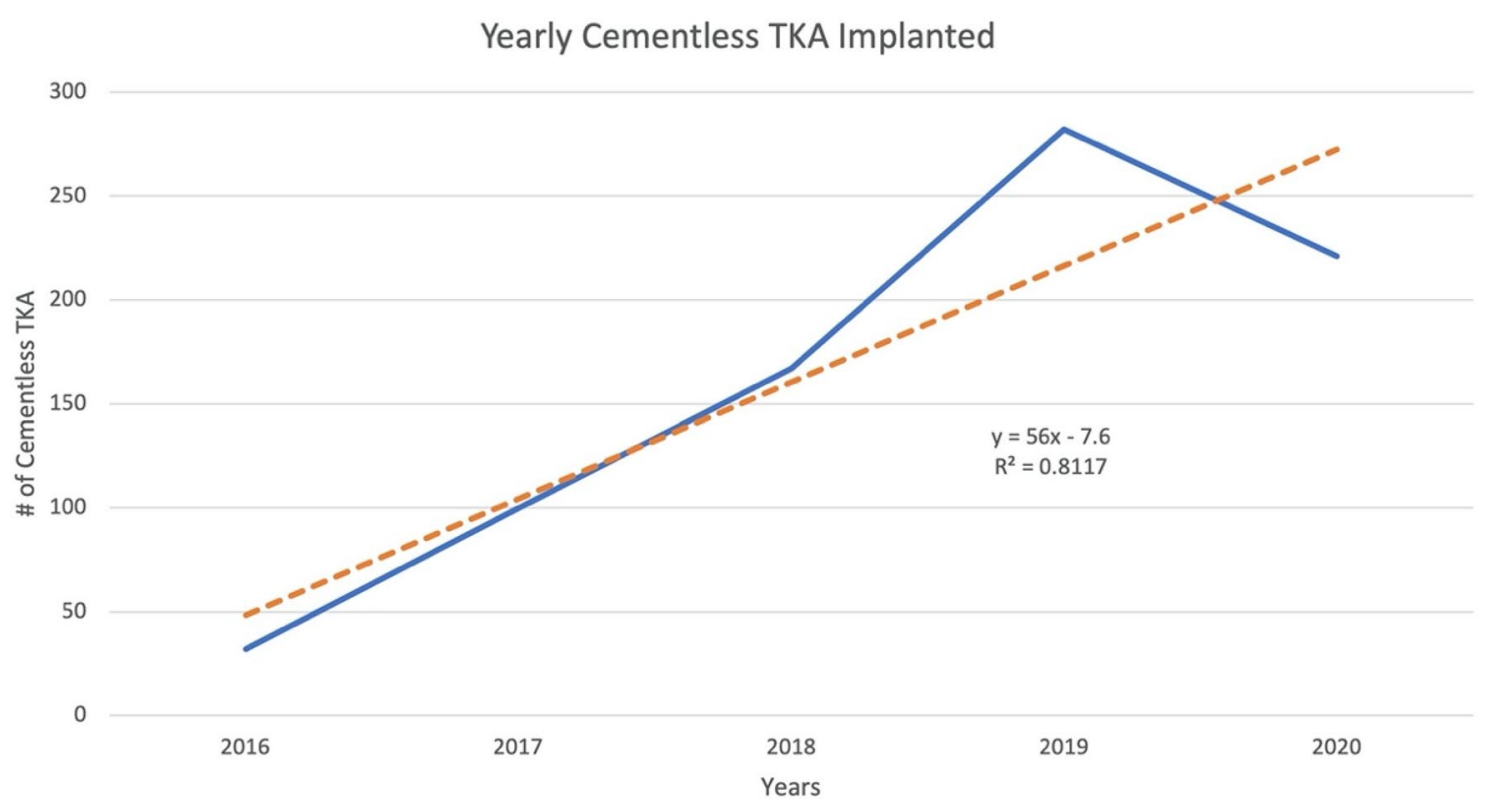

Fig. 1 Yearly utilization of cementless TKA implants and trend line

reported outcomes at two years. Miller et al. found similar results when performing a retrospective review of patients receiving the same implant design when comparing cemented vs cementless fixation, both groups had similar rates of complications $(P=0.9)$. The cementless group had 7 revisions, one revision for aseptic loosening $(0.5 \%)$. While the cemented group had 8 revisions, five for aseptic loosening $(2.5 \%)$.

There is paucity in the literature on studies reporting long-term outcomes on modern cementless TKA. Recently, Kim et al. reported on older generation cementless TKA, patients underwent bilateral TKA (one cemented \& one cementless) with a mean follow-up of 23.8 years. The 25 -year aseptic survivorship for both groups was $98 \%$, this is the longest follow-up of any study comparing cementless vs cemented TKA fixation [25]. There is still a need for more studies reporting on the long-term follow-up of cementless TKA fixation. Therefore, concerns regarding failure of fixation with cementless implants remain, there is a need for more long-term data and randomized clinical trials. Although changing clinical practice takes time the successful transition from cemented to cementless fixation has already occurred in THA procedures. As time passes technology and design will continue to improve, clearly the interest in this area is increasing and it is likely that the use of cementless TKA continues to grow.

Due to the retrospective nature of our study there are several limitations. (1) There is a limited follow up period for the most recently performed surgeries leading to immortal time bias. (2) We did not compare to cemented fixation or include functional outcomes as it was outside the scope of this study.

In conclusion, it is now clear as the data suggests that noncemented fixation in TKA is growing and here to stay. The more burning question is to what degree will its penetration be? Will it achieve $95 \%$ dominance as in the hip? It is the senior author's opinion that it is possible, but it still will require some tibial design changes to deal with osteoporotic bone especially seen on the Indian subcontinent.

\section{Declarations}

Conflict of interest Amar S Ranawat declares he has received IP royalties from DePuy and Stryker. Samuel Rodriguez declares no conflicts of interest.

Ethical Standard statement Ethical committee clearance was obtained from the Hospital for Special Surgery's Institutional Review Board.

Informed consent For this type of study informed consent is not required

\section{References}

1. Sloayn, M., Premkumar, A., \& Sheth, N. P. (2018). Projected volume of primary total joint arthroplasty in the U.S 2014 to 2030. Journal of Bone and Joint Surgery, 100, 1455-1460.

2. Ranawat, C. S., Meftah, M., Windsor, E. N., \& Ranawat, A. S. (2012). Cementless fixation in total knee arthroplasty: down the 
boulevard of broken dreams-affirms. Journal of Bone and Joint Surgery, 94, 82-84.

3. Nugent, M., Wyatt, M. C., Frampton, C. M., \& Hooper, G. J. (2019). Despite improved survivorship of uncemented fixation in total knee arthroplasty for osteoarthritis, cemented fixation remains the gold standard: an analysis of a national joint registry. Journal of Arthroplasty, 34, 1626-1633. https://doi.org/10.1016/j. arth.2019.03.047

4. Rodriguez, J. A., Bhende, H., \& Ranawat, C. S. (2001). Total condylar knee replacement: a 20-year follow-up study. Clinical Orthopaedics and Related Research, 388, 10-17.

5. American Joint Replacement Registry (AJRR). (2020). 2020 annual report. Rosemont, IL: American Academy of Orthopaedic Surgeons AAOS.

6. Vasarhelyi, E. M., \& Petis, S. M. (2020). Use of national joint registries to evaluate a new knee arthroplasty design. Journal of Arthroplasty, 35, 413-416. https://doi.org/10.1016/j.arth.2019.09. 018

7. Quispel, C. R., Duivenvoorden, T., Beekhuizen, S. R., Verburg, H., Spekenbrink-Spooren, A., Van Steenbergen, L. N., et al. (2020). Comparable mid-term revision rates of primary cemented and cementless total knee arthroplasties in 201,211 cases in the Dutch Arthroplasty Register (2007-2017). Knee Surgery and Sport Traumatology, Arthroscopy. https://doi.org/10.1007/ s00167-020-06183-2

8. Niemeläinen, M. J., Mäkelä, K. T., Robertsson, O., W-Dahl, A., Furnes, O., Fenstad, A. M., et al. (2020). The effect of fixation type on the survivorship of contemporary total knee arthroplasty in patients younger than 65 years of age: a register-based study of 115,177 knees in the Nordic Arthroplasty Register Association (NARA) 2000-2016. Acta Orthopaedica, 91, 184-190.

9. Hampton, C. B., Berliner, Z. P., Nguyen, J. T., Mendez, L., Smith, S. S., Joseph, A. D., et al. (2020). Aseptic loosening at the tibia in total knee arthroplasty: a function of cement mantle quality? Journal of Arthroplasty, 35, S190-S196.

10. Kurtz, S. M., Lau, E., Ong, K., Zhao, K., Kelly, M., \& Bozic, K. J. (2009). Future young patient demand for primary and revision joint replacement: national projections from 2010 to 2030. Clinical Orthopaedics and Related Research, 467, 2606-2612.

11. Julin, J., Jämsen, E., Puolakka, T., Konttinen, Y. T., \& Moilanen, T. (2010). Younger age increases the risk of early prosthesis failure following primary total knee replacement for osteoarthritis. A follow-up study of 32019 total knee replacements in the Finnish Arthroplasty Register. Acta Orthopaedica, 81, 413-419.

12. Murr, L. E., Gaytan, S. M., Martinez, E., Medina, F., \& Wicker, R. B. (2012). Next generation orthopaedic implants by additive manufacturing using electron beam melting. International Journal of Biomaterials, 2012, 245727.

13. Harwin, S. F., Patel, N. K., Chughtai, M., Khlopas, A., Ramkumar, P. N., Roche, M., et al. (2017). Outcomes of newer generation cementless total knee arthroplasty: beaded periapatite-coated vs highly porous titanium-coated implants. Journal of Arthroplasty, 32, 2156-2160. https://doi.org/10.1016/j.arth.2017.01.044
14. Van Hamersveld, K. T., Marang-Van De Mheen, P. J., Tsonaka, R., Valstar, E. R., \& Toksvig-Larsen, S. (2017). Fixation and clinical outcome of uncemented peri-apatite-coated versus cemented total knee arthroplasty: five-year follow-up of a randomised controlled trial, using radiostereometric analysis (RSA). Bone Joint Journal, 99B, 1467-1476.

15. Chong, D. Y. R., Hansen, U. N., van der Venne, R., Verdonschot, N., \& Amis, A. A. (2011). The influence of tibial component fixation techniques on resorption of supporting bone stock after total knee replacement. Journal of Biomechanics, 44, 948-954.

16. Nam, D., Lawrie, C. M., Salih, R., Nahhas, C. R., Barrack, R. L., $\&$ Nunley, R. M. (2019). Cemented versus cementless total knee arthroplasty of the same modern design. Journal of Bone Joint Surgery, 101, 1185-1192.

17. Lawrie, C. M., Schwabe, M., Nunley, R. M., Barrack, R. L., \& Pierce, A. (2019). The cost of implanting a cemented versus cementless total knee arthroplasty. Bone Joint Journal, 101, 61-63.

18. Bedard, N. A., Elkins, J. M., \& Brown, T. S. (2020). Effect of COVID-19 on hip and knee arthroplasty surgical volume in the United States. Journal of Arthroplasty, 35, S45-S48.

19. Berger, R. A., Lyon, J. H., Jacobs, J. J., Barden, R. M., Berkson, E. M., Sheinkop, M. B., et al. (2001). Problems with cementless total knee arthroplasty at 11 years follow-up. Clinical Orthopaedics and Related Research, 392, 196-207.

20. Rosenberg, A. G., Andriacchi, T. P., Barden, R., \& Galante, J. O. (1988). Patellar component failure in cementless total knee arthroplasty. Clinical Orthopaedics and Related Research, 236, 106-114.

21. Stulberg, S. D., Stulberg, B. N., Hamati, Y., \& Tsao, A. (1988). Failure mechanisms of metal-backed patellar components. Clinical Orthopaedics and Related Research, 236, 88-105.

22. Restrepo, S., Smith, E. B., \& Hozack, W. J. (2021). Excellent mid-term follow-up for a new 3D-printed cementless total knee arthroplasty. Bone Joint Journal, 103, 32-37.

23. Fricka, K. B., McAsey, C. J., \& Sritulanondha, S. (2019). To cement or not? Five-year results of a prospective, randomized study comparing cemented vs cementless total knee arthroplasty. Journal of Arthroplasty, 34, S183-S187. https://doi.org/10.1016/j. arth.2019.02.024

24. Mont, M. A., Gwam, C., Newman, J. M., Chughtai, M., Khlopas, A., Ramkumar, P. N., et al. (2017). Outcomes of a newer-generation cementless total knee arthroplasty design in patients less than 50 years of age. Ann Transl Med, 5, 1-6.

25. Kim, Y.-H., Park, J.-W., \& Jang, Y.-S. (2021). The 22 to 25 -year survival of cemented and cementless total knee arthroplasty in young patients. Journal of Arthroplasty, 36, 566-572.

Publisher's Note Springer Nature remains neutral with regard to jurisdictional claims in published maps and institutional affiliations. 